\title{
Randomised double blind trial of morphine versus diamorphine for sedation of preterm neonates
}

\author{
C M Wood, J A Rushforth, R Hartley, H Dean, J Wild, M I Levene
}

\begin{abstract}
Aims-To compare the safety and effectiveness of morphine and diamorphine for the sedation of ventilated preterm neonates in a double blind, randomised trial. Methods-Eighty eight babies were allocated to receive either morphine $(n=44)$ or diamorphine $(n=44)$ by bolus infusion (200 or $120 \mathrm{mcg} / \mathrm{kg}$, respectively, over two hours), followed by maintenance infusion (25 or $15 \mathrm{mcg} / \mathrm{kg} / \mathrm{h}$, respectively) during the initial phase of their respiratory disease. Serial monitoring of physiological, behavioural, and biochemical variables over the first 24 hours of the infusions was performed. Longer term outcomes were also monitored.

Results-Morphine, but not diamorphine, was associated with a mean (SEM) decrease in mean arterial blood pressure of $2.2(1.0) \mathrm{mm} \mathrm{Hg}(\mathrm{p}=0.05)$ over the initial loading infusion. Physiological (blood pressure variability) and behavioural measures of sedation (clinical assessment and sedation scoring) indicated that the two drug regimens were equally effective after 24 hours, but the sedative effects of diamorphine were evident more quickly than those of morphine. Both regimens significantly reduced plasma adrenaline concentrations over the first 24 hours of the infusions. No significant differences in mortality, ventilator days, chronic lung disease or intracranial lesions were noted.

Conclusions-Both drug regimens reduce the stress response to ventilation in preterm neonates. However, diamorphine's more rapid onset of sedation and morphine's hypotensive tendency suggest that diamorphine is preferable for the sedation of mechanically ventilated preterm neonates.
\end{abstract}

(Arch Dis Child Fetal Neonatal Ed 1998;79:F34-F39)

Academic Unit of Paediatrics and Child Health,

University of Leeds,

The General Infirmary

at Leeds

$\mathrm{C} M$ Wood

J A Rushforth

R Hartley

H Dean

J Wild

M I Levene

Correspondence to: Dr CM Wood

Department of Paediatrics, Hull Maternity Hospital,

Hedon Road,

Hull HU9 5LX.

Accepted 14 January 1998 reduction in the catecholamine stress response associated with the first 24 hours of IPPV.

We have studied the use of morphine in this situation before but noted two potential problems with its use. There was a trend towards a small but potentially important drop in blood pressure over the initial six hours of the morphine infusion. ${ }^{45} \mathrm{~A}$ proportion of babies also continue to struggle against the ventilator despite the drug treatment and may require further measures such as neuromuscular paralysis in the initial phases of their illness. ${ }^{3}$

Diamorphine (3,6-diacetylmorphine) is more lipid soluble than morphine and this property has been considered responsible for the perception that diamorphine works more quickly than morphine. ${ }^{6}$ It is also associated with fewer hypotensive effects than morphine, probably by virtue of reducing the amount of histamine released. We hypothesised that diamorphine may be more effective than morphine at achieving early, effective sedation in preterm babies, while not causing any more adverse side effects than the drug in current use. Pharmacokinetic data from a diamorphine infusion regimen used for preterm neonates showed that serum morphine concentrations thought to be appropriate for analgesia in these young infants could be achieved. ${ }^{7}$ We compared the safety and efficacy of a diamorphine infusion regimen based on their pharmacokinetic data with our standard morphine regimen for the sedation of ventilated premature neonates in a double blind, randomised, controlled trial.

\section{Methods}

The effects of the two drug regimens were studied in premature babies admitted to the neonatal intensive care unit at the Leeds General Infirmary over 18 months. Babies were eligible for entry to the trial if they were less than 35 weeks of postconceptional age at birth, greater than 2 hours but less than 48 hours old at trial entry, and required IPPV and therefore sedation under our prevailing protocol. Further requirements were the establishment of indwelling intra-arterial access and informed parental consent for the infants to be entered into the trial. A requirement for early neonatal surgery, severe congenital malformation, or the previous administration of opiates to the baby were exclusion criteria.

Babies admitted to the trial were randomly allocated to receive either morphine (200 $\mathrm{mcg} / \mathrm{kg}$ loading dose over two hours, followed by maintenance infusion of $25 \mathrm{mcg} / \mathrm{kg} / \mathrm{h}$ ) or diamorphine $(120 \mathrm{mcg} / \mathrm{kg}$ over 2 hours and then $15 \mathrm{mcg} / \mathrm{kg} / \mathrm{h}$ ). Infusion solutions were made up by the hospital pharmacy in identically presented syringes containing either 2.5 $\mathrm{mg}$ morphine or $1.5 \mathrm{mg}$ diamorphine in $50 \mathrm{ml}$ 
Table 1 Patient details at trial entry: (median + interquartile range)

\begin{tabular}{llll}
\hline & \multicolumn{2}{l}{ Treatment group } & \\
\cline { 2 - 4 } & Morphine $(n=44)$ & Diamorphine $(n=44)$ & \\
\hline Postconceptional age (weeks) & $28(26-30)$ & $27(26-29)$ & $\mathrm{p}=0.34$ \\
Birthweight (g) & $1058(810-1440)$ & $980(830-1270)$ & $\mathrm{p}=0.45$ \\
Sex ratio $(\mathrm{M} / \mathrm{F})$ & $28 / 16$ & $24 / 20$ & $\mathrm{p}=0.39$ \\
A:a ratio & $0.24(0.14-0.57)$ & $0.39(0.16-0.57)$ & $\mathrm{p}=0.40$ \\
FIO & $0.45(0.3-0.7)$ & $0.35(0.21-0.7)$ & $\mathrm{p}=0.16$ \\
Received surfactant & 35 & 32 & $\mathrm{p}=0.45$ \\
Mean arterial blood pressure (mm Hg) & $34(29-38)$ & $35(32-40)$ & $\mathrm{p}=0.59$ \\
\hline
\end{tabular}

Table 2 Clinical outcomes: median (IQR) or number (\%)

\begin{tabular}{lccc}
\hline \multicolumn{4}{c}{ Treatment group } \\
\cline { 2 - 4 } & Morphine $(n=44)$ & Diamorphine $(n=44)$ & \\
\hline Duration of infusion (h) & $39(21-67)$ & $25(18-67)$ & $\mathrm{p}=0.38$ \\
Days ventilated & $4(3-11)$ & $4(2.5-9)$ & $\mathrm{p}=0.56$ \\
IVH (all grades) & $15(34 \%)$ & $23(52 \%)$ & $\mathrm{p}=0.09$ \\
Parenchymal brain lesions & $8(18 \%)$ & $5(11 \%)$ & $\mathrm{p}=0.37$ \\
Air leak & $5(11 \%)$ & $4(9 \%)$ & $\mathrm{p}=0.73$ \\
Patent ductus arteriosus & $9(20 \%)$ & $12(27 \%)$ & $\mathrm{p}=0.45$ \\
$\mathrm{O}_{2}$ required at 28 days & $16(36 \%)$ & $15(34 \%)$ & $\mathrm{p}=0.82$ \\
Death (up to 28 days) & $7(16 \%)$ & $6(14 \%)$ & $\mathrm{p}=0.76$ \\
\hline
\end{tabular}

(IQR = interquartile range)

$10 \% \mathrm{w} / \mathrm{v}$ dextrose solution. Clinicians, nurses, and parents were blinded to the randomisation. The decision to discontinue the opiate infusions was taken by the clinical team caring for the baby and was based on clinical grounds $\left(\mathrm{FIO}_{2}<0.4\right.$, peak inspiratory pressure $<20 \mathrm{~cm}$ $\mathrm{H}_{2} \mathrm{O}$, IMV rate $<20 / \mathrm{min}$ ).

Babies were continuously monitored for arterial blood pressure, heart rate, $\mathrm{PaO}_{2}$ (or $\mathrm{SaO}_{2}$ ) and temperature. Intermittent arterial blood gas sampling was performed, as clinically appropriate, to determine ventilatory requirements. Interventions to support the circulation were made on clinical grounds and followed the protocol for treatment of hypotension in use on our unit. Mean arterial blood pressure (MABP) was recorded when starting the infusion, and at 2 (at the end of the loading infusion), 6 , and 24 hours. The use or initiation of inotropic support over the first 24 hours of the infusion was also recorded.

The sedation achieved by the drug infusion was assessed in three ways. First, we assessed the beat to beat variability of the arterial blood pressure (ABP) trace as a measure of the babies interaction with the ventilator. This variability, as determined by the coefficient of variation (CV) of 10 successive systolic blood pressure values, was taken to reflect the variable changes of intrathoracic pressure associated with asynchronous interaction between the baby and the ventilator. ${ }^{2}$ Secondly, the nurse looking after the baby completed a four parameter sedation score (Appendix 1) and recorded a qualitative judgment of successful or unsuccessful sedation. Study babies who had received neuromuscular paralysis were not included in the analysis of sedation scores. Third, plasma concentrations of adrenaline and noradrenaline were measured in arterial blood samples. Samples were immediately centrifuged, the plasma separated and stored at $-20^{\circ} \mathrm{C}$ until analysed using a radioenzymatic technique. ${ }^{89}$ Samples of plasma were also collected for measurement of drug and metabolite concentrations at set times over the first 60 hours of the infusions, not more than four samples being collected from any one baby. Blood pressure variability and nurse assessment of the sedation score was recorded at $0,2,6$ and 24 hours into the infusion. Blood samples for catecholamine concentrations were collected at 0 and 24 hours.

Results were analysed using the MannWhitney U test or Wilcoxon signed rank test for non-parametric unpaired or paired data, respectively. The paired $t$ test was used to analyse changes in mean $\mathrm{ABP}$ within groups compared with baseline values. Categorical comparisons between groups were tested using the $\chi^{2}$ test with Yates' correction where appropriate. Statistical analysis was performed using the Minitab program. Approval for the study was obtained from the local hospital ethical committee.

\section{Results}

There were no significant differences between the two groups on entry into the study (table 1). Similar numbers in each group had received surfactant (Curosurf) for respiratory distress syndrome before trial entry. Other diagnoses (apnoea, pneumonia, and asphyxia) were evenly spread between the groups. There were no significant differences between the two groups of babies in terms of the major short and longer term outcome measures listed in table 2 . The median (interquartile range) duration of infusion for the babies who received morphine was 39 (21-67) hours compared with 25 (18-67) hours for the diamorphine group, but the median number of days on the ventilator was identical (4 days) for the two groups.

\section{EFFECTS ON BLOOD PRESSURE}

Both groups of babies had similar mean ABP at the start of opiate infusion (fig 1). Three babies from each group had required dopamine support before starting the infusion. Mean ABP fell in the morphine treated group over the first two hours (loading infusion) by a mean (SEM) of $2.2(1.0) \mathrm{mm} \mathrm{Hg}(\mathrm{p}=0.05)$. In contrast, the mean ABP of babies treated with diamorphine did not show any significant change over the same period, rising by $1.2(0.7)$ $\mathrm{mm} \mathrm{Hg}(\mathrm{p}=0.3)$. After the initial loading infusion both groups then showed gradual increases in mean ABP over the first 24 hours (fig 1). Of the 44 babies receiving diamorphine, $14(32 \%)$ required dopamine to support the

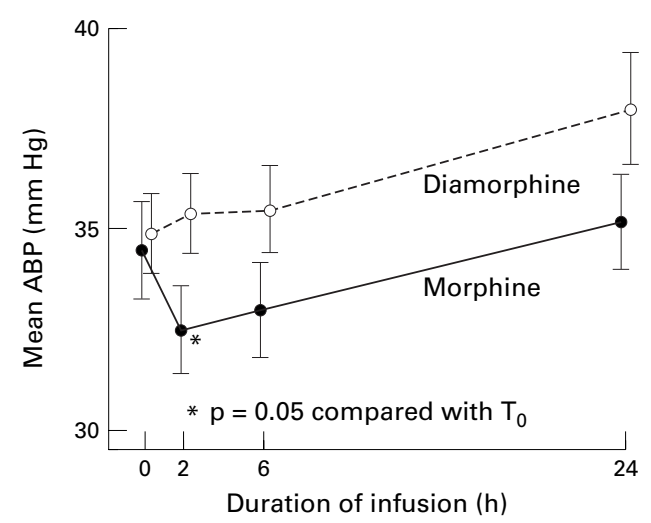

Figure 1 Mean (SEM) changes in MAP over the first 24 hours of morphine and diamorphine infusions. 


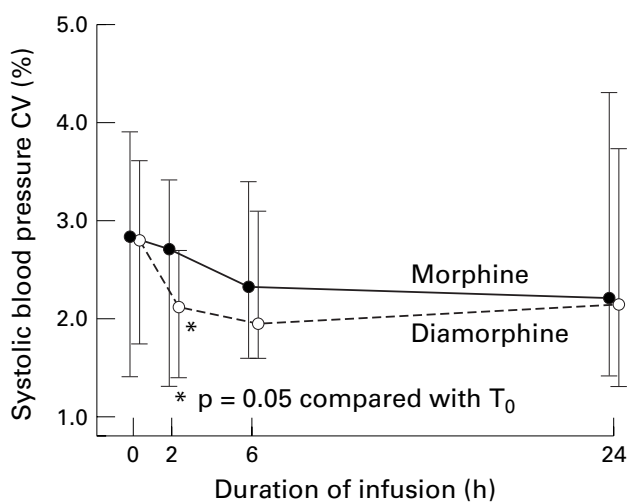

Figure 2 Changes in the coefficient of variation in systolic blood pressure (median and interquartile range) over the first 24 hours of morphine and diamorphine infusions.

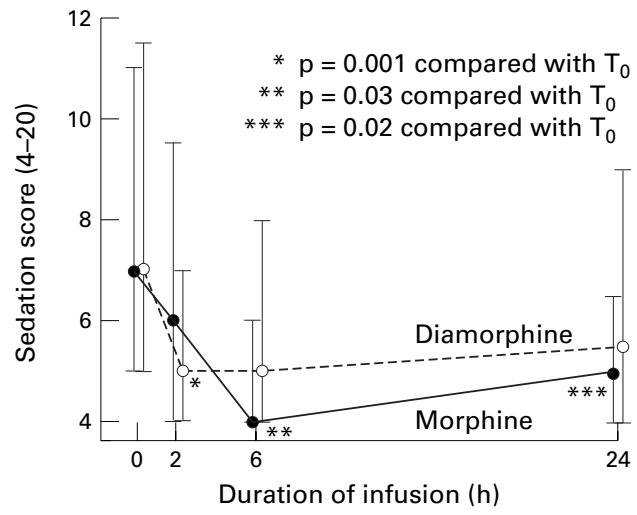

Figure 3 Changes in sedation scores from non-paralysed babies (median and interquartile range) over the first 24 hours of morphine and diamorphine infusions.

$\mathrm{ABP}$ at some time over the first 24 hours of the infusion, compared with $20(45 \%)$ out of the 44 receiving morphine (NS).

Blood pressure variability data were available for 45 babies ( 23 morphine, 22 diamorphine). The median variability of the arterial systolic pressure was similar in the two groups at the start of opiate infusion (morphine $=2.8 \%$, diamorphine $=2.9 \%$ ) (fig 2). For the diamorphine group the $\mathrm{CV}$ of the systolic blood pressure reduced significantly over the period of the loading infusion $(\mathrm{p}=0.05$, Wilcoxon signed rank test) and then remained constant over the first 24 hours. This effect was only gradually achieved in the morphine treated group, although differences between the two groups at any one time were not significant and both groups showed similar median blood pressure variability after 24 hours (morphine $=2.3 \%$, diamorphine $=2.2 \%$ ).

CLINICAL ASSESSMENT OF SEDATION

Both groups of babies showed a similar degree of sedation at the start of the opiate infusion as assessed clinically using the sedation score (fig $3)$. In the 44 babies for whom clinical sedation scores were available (18 morphine, 26 diamorphine) there was a significant decrease in the score over the first two hours for the babies receiving diamorphine (median value decreasing from 7 to $5, p=0.001)$. No significant decrease in sedation score was observed in the babies receiving morphine until six hours after starting the infusion (median value decreasing from 7 to $4, \mathrm{p}=0.03$ ) and the score remained significantly lower than baseline at 24 hours. The difference in sedation scores between the two groups was not significant at any stage over the first 24 hours of the infusions. A clinical decision to start pancuronium during this period was taken for eight of the babies who received morphine (median time after starting the infusion was 5 hours) compared with seven of the babies who received diamorphine (median time 9.5 hours). For those babies who were not paralysed, the attending nurses assessed the degree of sedation to be adequate on clinical grounds at some point over the first 24 hours of the opiate infusion in $19(66 \%)$ of 29 babies receiving morphine and 15 (52\%) of 29 receiving diamorphine (NS).

EFFECTS ON CATECHOLAMINES

Paired catecholamine data are available for 37 babies (17 morphine, 20 diamorphine). Babies who were receiving dopamine before the start of the infusion or who started dopamine or other inotropes during the first 24 hours of the infusion were not included in the paired analysis due to interference by exogenous dopamine with the assay. Babies who did not complete 24 hours of sedation (sedation stopped or baby died) did not have catecholamines measured at 24 hours and were therefore also excluded from the paired analysis. Plasma adrenaline and noradrenaline concentrations were comparable between the two groups at the start of infusions (table 3). Significant decreases in the adrenaline concentration over the first 24 hours of the infusion were noted for both groups, but although noradrenaline concentrations fell significantly in the morphine group, there was no significant change in noradrenaline concentrations in those babies treated with diamorphine (table 3) (fig 4).

CLINICAL OUTCOME MEASURES

No significant differences were noted for the clinical outcomes listed in table 2.The incidence of intraventricular haemorrhage (IVH) was higher in the diamorphine group (52\%) than in the morphine group (34\%), but the incidence of parenchymal lesions was higher in the morphine group (18\% compared with $11 \%)$. The incidence of air leaks, clinically significant patent

Table 3 Plasma catecholamine values (nmol/l)

\begin{tabular}{lcrl}
\hline & \multicolumn{2}{l}{ Treatment group } & \\
\cline { 2 - 4 } & Morphine $(n=17)$ & Diamorphine $(n=20)$ & $p(M W U$ test $)$ \\
\hline Plasma adrenaline at $\mathrm{T}_{0}$ (median $\left.+\mathrm{IQR}\right)$ & $1.48(0.59-3.74)$ & $1.08(0.73-2.67)$ & 0.95 \\
Median change in plasma adrenaline $\mathrm{T}_{0}-\mathrm{T}_{24}(\mathrm{p}$ value) & $-0.93(0.001)$ & $-0.58(0.001)$ & 0.34 \\
Plasma noradrenaline at $\mathrm{T}_{0}$ (median $\left.+\mathrm{IQR}\right)$ & $3.29(2.90-6.83)$ & $3.86(1.89-7.81)$ & 0.55 \\
Median change in plasma noradrenaline $\mathrm{T}_{0}-\mathrm{T}_{24}(\mathrm{p}$ value) & $-2.07(0.005)$ & $-0.62(0.20)$ & 0.13 \\
\hline
\end{tabular}

$\mathrm{IQR}=$ interquartile range; $\mathrm{T}_{0}=$ time of starting infusion; $\mathrm{T}_{24}=24 \mathrm{~h}$ after starting infusion 

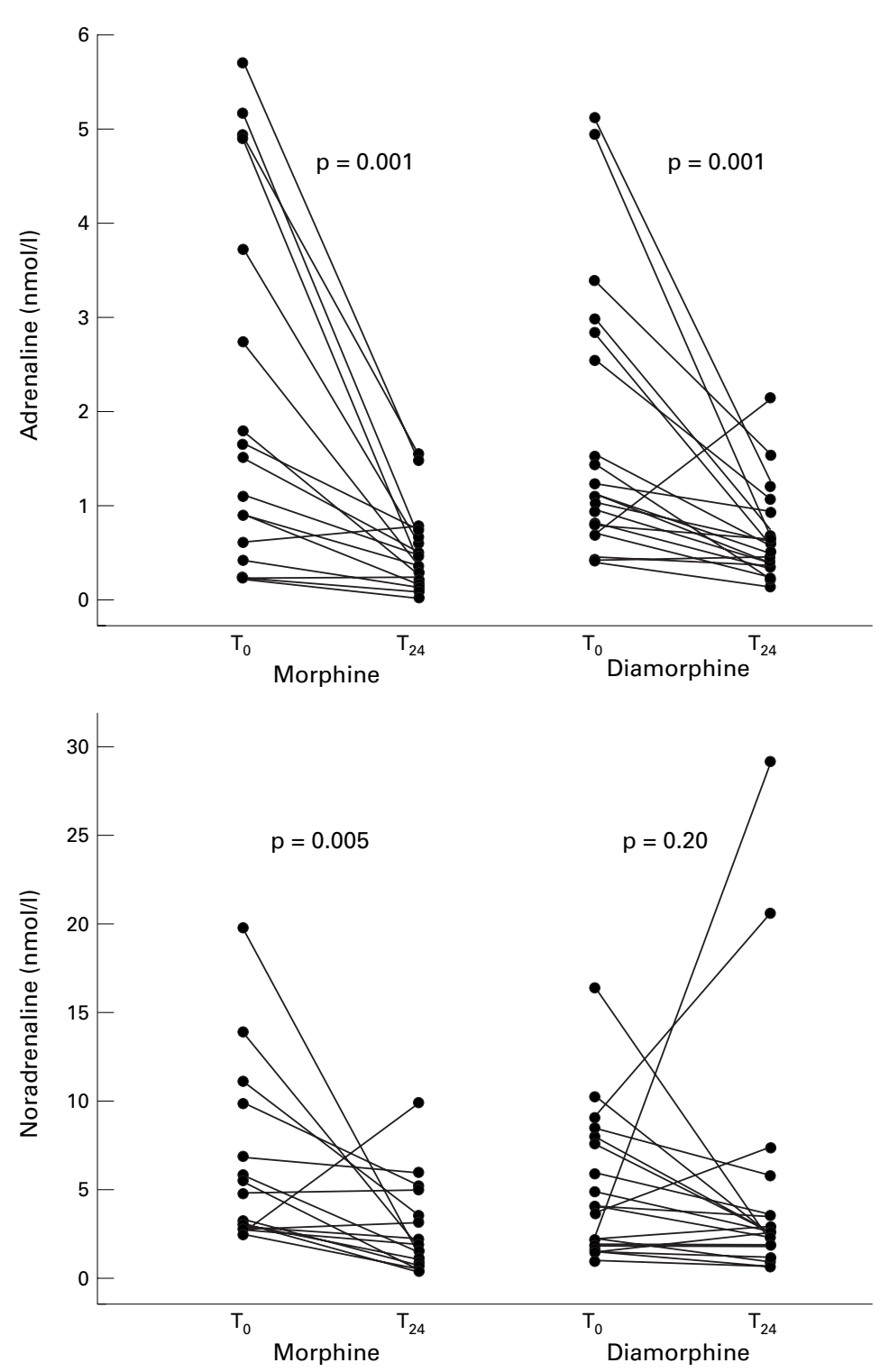

Figure 4 Changes in plasma adrenaline and noradrenaline over the first 24 hours of morphine and diamorphine infusions.

ductus arteriosus, requirements for oxygen at 28 days of life and mortality were similar between the two groups of babies.

\section{Discussion}

The effects of morphine in preterm babies have been documented before, showing that sedation can be achieved effectively by using a loading infusion over two hours, followed by a maintenance infusion. ${ }^{4}$ Sedation can be assessed by measuring physiological variables, ${ }^{10}$ biochemical parameters such as the stress hormones (catecholamines, cortisol), ${ }^{11}$ and by behavioural measures (clinical observation and scoring systems). ${ }^{12}$ We compared the effectiveness of morphine with that of a theoretically faster acting opiate, diamorphine, in the context of sedating the ventilated preterm neonate using physiological (ABP variability), biochemical (catecholamines), and behavioural measures of the degree of sedation achieved by the two drugs.

The safety of the two drugs in this population was a prime concern as previous studies had

\section{Key points}

- Both morphine and diamorphine sedate preterm babies undergoing intensive care effectively and to an equal extent over the first 24 hours of infusion.

- Diamorphine has a more rapid onset of sedation than morphine.

- Morphine is associated with an initial hypotensive effect which is not seen with diamorphine.

suggested a trend towards a hypotensive effect associated with the use of morphine. ${ }^{4}$ In adults and older children this effect of boluses of morphine is well recognised and is said to be related to histamine release. We confirmed a small but significant drop in ABP associated with the administration of the loading infusion of morphine over the first two hours. We did not see this effect in association with the use of diamorphine given by an infusion regimen designed to give a clinically comparable sedative effect based on previously published studies. A small but significant fall in blood pressure has been reported using diamorphine before, but that was associated with either a 50 or 200 $\mathrm{mcg} / \mathrm{kg}$ loading dose given over 30 minutes. ${ }^{13}$ In our study a $120 \mathrm{mcg} / \mathrm{kg}$ loading dose over 2 hours did not have any significant hypotensive effect and we calculate that this study had $>90 \%$ power to detect a $10 \%(3.5 \mathrm{~mm} \mathrm{Hg})$ change in blood pressure for this group at a $5 \%$ significance level. More of the babies who received morphine also required inotropic support over the first 24 hours of the infusion and more developed parenchymal brain lesions on ultrasound scanning than did those who received diamorphine, although these differences between the groups were not significant. The mean decrease in ABP was small, but the addition of a drug which lowers the ABP in babies at risk of hypotension and in whom decreases in cerebral perfusion pressure may cause major long term lesions can only be justified if it gives clinically significant benefits. Equally, if a similar drug can achieve the same (or better) clinical effect without the same potentially hazardous side effects then it should be the drug of choice.

The use of morphine to sedate preterm babies receiving IPPV is based on its effects as a respiratory depressant, and on its analgesic and psychotropic actions. Babies may interact with the mechanical ventilator in a potentially harmful manner and one aim of using morphine is to reduce this interaction. There is good evidence that morphine can reduce the stress associated with the experience of neonatal intensive care, but a proportion of babies continue to interact strenuously against the ventilator and require other measures to reduce this. ${ }^{3}$ Using the variability of the arterial systolic blood pressure as a marker for interaction between the ventilator and the baby's own respiratory activity, we have been able to show that diamorphine infusion significantly decreased variability over the first two hours of infusion. This effect was not noted in association with the use of morphine 
until 6 hours into the infusion. This more rapid pharmacodynamic effect of diamorphine might be expected on the basis of its pharmacokinetics as it is a more lipid soluble drug. It can therefore penetrate tissues such as the blood-brain barrier more quickly than morphine, enabling more rapid access to the central nervous system opiate receptors by its deacetylated active metabolites. ${ }^{6}$ It is also perceived to be a faster acting opiate than morphine in older children and adults. Its higher lipid solubility would also increase uptake into the liver, suggesting that conversion to active glucuronide metabolites might be enhanced compared with morphine. The pharmacokinetics of these two drug regimens will be the subject of a further paper, which may help to explain the observed pharmacodynamic differences between them.

The clinical impression of the degree of sedation achieved, often assessed by the nursing staff looking after the baby, is the most widely used criterion on which decisions to alter treatment strategies are based. These aim to reduce potentially harmful respiratory interaction against the ventilator. The use of clinical scoring systems can quantify this otherwise rather subjective measure and we asked the nursing staff looking after the babies to use a four parameter behavioural score (consciousness level, crying, posturing and facial expression) to assess each baby's level of sedation at four points over the initial 24 hours of the opiate infusions. A maximum score of 20 would indicate very poor sedation whereas a minimum score of 4 would indicate a heavily sedated and largely unresponsive child. Unfortunately, we were not able to collect complete scores for all babies in the study, emphasising that the addition of a further item of information for already busy nurses to record may result in a less than complete data set. Among those babies for whom data were available, however, we found that both groups scored in the mid range when they were entered into the study. The babies treated with diamorphine were significantly more sedated by the time the loading infusion had finished than they had been at the start of the infusion. This effect was not seen in the babies treated with morphine until six hours, but by this stage at least half of the babies for whom the data were available in this group were heavily sedated with clinical scores of 4 . We do not know the ideal score that we should be aiming to achieve, but suggest that if babies are so heavily sedated as to be unresponsive then excessive sedation is being used. Both groups were felt to show similar levels of sedation over the first 24 hours, according to the staff looking after them. A small proportion in both groups continued to struggle against the ventilator, but although similar numbers in the two groups of babies were judged to require neuromuscular paralysis with pancuronium, this decision tended to be made earlier in the course of the infusion for the morphine babies than for those treated with diamorphine. This again suggests that sedation with diamorphine may be more effective in the early stages of the treatment than if morphine is used.

We have already shown that morphine infusion can reduce the plasma concentrations of catecholamine stress hormones compared with placebo. ${ }^{4}$ Similar reductions in adrenaline and noradrenaline have recently been reported with the use of diamorphine, although two different doses of diamorphine seemed to have differing effects on the two hormones. ${ }^{13}$ We have confirmed that diamorphine and morphine infusions both significantly reduce the plasma concentration of adrenaline over the first 24 hours of infusion at the doses used. This occurs after the initial physiological postnatal decrease in catecholamines seen in the first two hours of life when plasma concentrations in control subjects would be expected to remain constant or to increase. ${ }^{4}$ The results for plasma concentrations of noradrenaline were not as striking for either group, although a significant decrease was noted in the morphine group. The decreases in catecholamines observed showed no significant differences between the two groups, indicating that over 24 hours both drugs were equally effective sedatives as assessed on biochemical indices.

In a controlled, randomised, and double blinded comparison of the use of morphine or diamorphine infusions for the sedation of preterm neonates requiring IPPV, we showed that diamorphine is not associated with the small but significant hypotensive effect seen with morphine. It is as effective at achieving adequate sedation as morphine, when judged on physiological, behavioural, and biochemical measures. Its sedative effects also become clinically apparent more quickly than those of morphine. On the basis of the data available, the faster onset of action and reduced side effects associated with diamorphine make it a more logical choice for neonatal sedation than morphine.

1 Greenough A, Morley CJ, Wood S, Davis JA. Pancuronium prevents pneumothoraces in ventilated premature babies who actively expire against positive pressure inflation. Lancet $1984 ; \mathbf{i}: 1-3$

2 Perlman JM, McMenamin JB, Volpe JJ. Fluctuating cerebral blood-flow velocity in respiratory distress syndrome - relation to the development of intraventricular haemorrhage. N Engl f Med 1983;309:204-9.

3 Quinn MW, Otoo F, Rushforth JA, et al. Effect of morphine and pancuronium on the stress responses in ventilated preterm infants. Early Hum Dev 1992;30:241-8.

4 Quinn MW, Wild J, Dean HG, et al. Randomised double-blind controlled trial of effect of morphine on catecholamine concentrations in ventilated preterm babies. Lancet 1993;342:324-7.

5 Hartley R, Green M, Quinn MW, Levene MI. Pharmacokinetics of morphine infusion in premature neonates. Arch Dis Child 1993;69:55-8.

6 Hartwig P, Bergstrom K, Lindberg P, et al. Kinetics of ${ }^{11} \mathrm{C}-$ labelled opiates in the brain of rhesus monkeys. $f$ Pharm Exp Ther 1984; 230:250-5.

7 Elias-Jones AC, Barrett DA, Rutter N, Shaw PN, Davis SS. Diamorphine infusion in the preterm neonate. Arch Dis Child 1991;66:1155-7.

8 Morrison JFJ, Pearson SB, Dean HG. Parasympathetic nervous system in nocturnal asthma. BMf 1988;296:1427-9.

9 da Prada M, Zurcher G. Simultaneous radioenzymatic determination of plasma and tissue adrenaline, noradrenaline and dopamine within the femtomole range. Life Sci 1974;19:1161-74.

10 Owens ME, Todt EN. Pain in infancy: neonatal reaction to a heel lance. Pain 1984;20:213-30.

11 Anand KJS, Brown NJ, Bloom SR, Aynsley-Green A. Studies on the hormonal regulation of fuel metabolism in the human newborn infant undergoing anaesthesia and surgery. Hormone Res 1985;22:115-28.

12 Grunau RVE, Craig KD. Pain expression in neonates: facial action and cry. Pain 1987;28: 395-400.

13 Barker DP, Simpson J, Pawula M, Barrett DA, Shaw PN, Rutter N. Randomised double-blind trial of two loading dose regimens of diamorphine in ventilated newborn infants. Arch Dis Child 1995;73:F22-6. 
Appendix 1

Clinical sedation score

Choose the most appropriate description of the infant from each of the following. Add up the total score. Score 0 at any time that the infant is paralysed.

Conscious level:

1 Asleep

2 Eyes closed, small body movements

3 Eyes closed, large body movements

4 Eyes open occasionally

5 Fully alert

\section{Crying:}

1 No sign of attempt to cry

2 Trying to make sounds but not distressed

3 Trying to make sounds, appears distressed

4 Appears to be crying

5 Appears to be screaming
Posturing:

1 No movement

2 Moving fingers/toes only

3 Moving hands and feet only

4 Moving limbs (slowly but not a lot)

5 Moving limbs (quickly and vigorously)

Facial expression:

1 No movement

2 Movement but not distressed

3 Movement and distressed (eg brow furrow)

4 Partial grimace (2 out of: furrowed brow, screwed up eyes, tight cheek muscles)

5 Full grimace (all 3 expressions in 4 above) 